\title{
Matrix-Stiffness-Regulated Inverse Expression of Krüppel-Like Factor 5 and Krüppel-Like Factor 4 in the Pathogenesis of Renal Fibrosis
}

\author{
Wan-Chun Chen, ${ }^{*}$ Hsi-Hui Lin, ${ }^{\dagger}$ and Ming-Jer Tang ${ }^{* \dagger}$
}

From the Institute of Basic Medical Sciences* and the Department of Physiology, ${ }^{\dagger}$ National Cheng-Kung University Medical College, Tainan, Taiwan

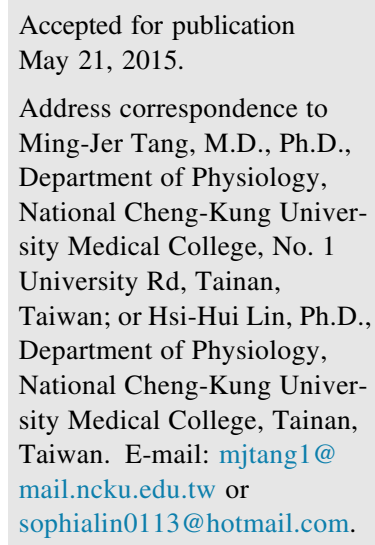

Address correspondence to Ming-Jer Tang, M.D., Ph.D., Department of Physiology, National Cheng-Kung University Medical College, No. 1 University Rd, Tainan, Taiwan; or Hsi-Hui Lin, Ph.D., Department of Physiology, National Cheng-Kung University Medical College, Tainan, Taiwan. E-mail: mjtang1@ mail.ncku.edu.tw or sophialin0113@hotmail.com.

\begin{abstract}
The proliferation of mouse proximal tubular epithelial cells in ex vivo culture depends on matrix stiffness. Combined analysis of the microarray and experimental data revealed that Krüppel-like factor (Klf) 5 was the most up-regulated transcription factor accompanied by the down-regulation of Klf4 when cells were on stiff matrix. These changes were reversed by soft matrix via extracellular signal-regulated kinase (ERK) inactivation. Knockdown of Klf5 or forced expression of Klf4 inhibited stiff matrix-induced cell spreading and proliferation, suggesting that $\mathrm{Klf5} / \mathrm{Klf} 4$ act as positive and negative regulators, respectively. Moreover, stiff matrix-activated ERK increased the protein level and nuclear translocation of mechanosensitive Yes-associated protein 1 (YAP1), which is reported to prevent Klf5 degradation. Finally, in vivo model of unilateral ureteral obstruction revealed that matrix stiffness-regulated Klf5/Klf4 is related to the pathogenesis of renal fibrosis. In the dilated tubules of obstructed kidney, ERK/YAP1/KLF5/cyclin D1 axis was up-regulated and Klf4 was down-regulated. Inhibition of collagen crosslinking by lysyl oxidase inhibitor alleviated unilateral ureteral obstruction-induced tubular dilatation and proliferation, preserved Klf4, and suppressed the ERK/YAP1/Klf5/cyclin D1 axis. This study unravels a novel mechanism how matrix stiffness regulates cellular proliferation and highlights the importance of matrix stiffnessmodulated Klf5/Klf4 in the regulation of renal physiologic functions and fibrosis progression. (Am J Pathol 2015, 185: 2468-2481; http://dx.doi.org/10.1016/j.ajpath.2015.05.019)
\end{abstract}

Renal fibrosis, a common pathologic condition in progressive chronic kidney disease, is characterized by excessive crosslinking or deposition of the extracellular matrix, particularly of collagenous fibers. With the use of the unilateral ureteral obstruction (UUO) model, we found that the fibrotic kidney was stiffer than the normal kidney (Y.C. Yeh, unpublished data). Accumulated data indicate that matrix stiffness, one of the mechanical forces acting on cells, has a large impact as chemical stimuli on the regulation of cell proliferation, apoptosis, and differentiation. $^{1-4}$ The maintenance of tissue stiffness is thus critical for the physiologic function of the organs. Disturbance of tissue stiffness interferes with tissue development and promotes disease progression. ${ }^{5}$ Tissue stiffening is now used as a disease marker for scleroderma, ${ }^{6}$ atherosclerosis, ${ }^{7}$ cancer, ${ }^{8}$ and fibrosis. ${ }^{9,10}$

Proximal tubules (PTs), the major part of the kidney, are responsible for reabsorption. During fibrosis, PT cells undergo a loss of apical-basal polarity, epithelial-mesenchymal transition
(EMT), and uncontrolled proliferation. Considering the importance of matrix stiffness in regulating cellular behavior and tissue function, we speculate whether increasing stiffness in the microenvironment is a prerequisite for the fibrotic response of PTs. With the use of the ex vivo primary culture system and matrices with tunable stiffness, we found that soft matrix retained primary mice PT epithelial cells (mPTECs) at a tubular-like structural characteristics with differentiated phenotypes and growth arrest. EMT induced by transformation growth factor- $\beta 1$, a key mediator in renal fibrosis, ${ }^{11}$ is also inhibited by soft matrix. ${ }^{12}$ In the present work, we studied the detailed mechanisms underlying the matrix stiffness-modulated behavior of mPTECs, particularly in relation to proliferation.

Supported by National Science Council grant NSC101-2320-B-006-011MY3 and Ministry of Science and Technology grant MOST103-2320-B006-044-MY3 (M.-J.T.).

Disclosures: None declared. 
On the basis of the microarray profiling of mPTECs on culture dishes, we found that a subfamily of Krüppel-like factors (Klfs) was markedly altered during culture (Table 1). Klfs, highly conserved zinc finger-containing transcription factors (TFs), are key regulators of cellular functions. To date, $17 \mathrm{Klfs}$ have been identified. ${ }^{13}$ Among these, the expressions of Klf4 and Klf5 are highly restricted in the epithelium of several organs. Klf4 and Klf5 antagonize each other by physical competition in controlling expression of target genes, which are related to proliferation, differentiation, apoptosis, development, and disease. ${ }^{14-16}$ Notably, our microarray data found that Klf5 was the most significantly up-regulated TF with the decrease of Klf4 during ex vivo culture (Table 1). In this study, we clarify the role of the inverse expression pattern of Klf5 and Klf4 in MPTECs.

Klf5 is highly expressed in proliferating epithelial cells during development and in adult tissues. ${ }^{15,17}$ Constitutive expression of Klf5 results in hyperplasia and a transformed phenotype by increasing cell cycle-related genes [cyclin B1 (Ccnbl), cyclin D1 (Ccndl), and cyclin-dependent kinase 1 $(C d k 1)]$ in both fibroblasts and epithelial cells, indicating that Klf5 functions as an oncogene. ${ }^{18-20}$ Conversely, Klf4 is known to regulate the terminal differentiation of the epithelium in several organs, such as the gut, skin, and mammary gland. ${ }^{21-24}$ The early lethality of Klf4-null mice indicates the defective epithelial barrier function, which leads to hydration. ${ }^{25}$ Klf4 maintains the epithelial phenotype and prevents EMT. ${ }^{26}$ Inducible expression of Klf4 blocks cell cycle by inhibiting cyclin D1 in both normal and cancer cells, suggesting that Klf4 functions as a tumor suppressor. ${ }^{15,27-29}$ In addition, Klf4 is essential for maintaining cancer stem cells or stem cells. ${ }^{30,31}$ Collectively, Klf5 and Klf4 are inversely expressed and exert opposite effects on differentiation and proliferation: Klf5 stimulates proliferation, whereas Klf4 promotes cell differentiation.

In kidneys, Klf5 is only expressed in the collecting duct epithelium and is increased for the initiation and progression of inflammatory responses after UUO. ${ }^{32}$ Klf4 contributes to nephron differentiation in embryonic kidneys. ${ }^{33,34}$ Klf4 in glomerular podocytes facilitates cell function and a reduction in proteinuria. ${ }^{35}$ Here, we report that inverse expression of Klf5 and Klf4 was switched between soft and stiff matrices. Yes-associated protein 1 (YAP1)-transduced mechanical cues from matrix stiffness may regulate the inverse expression of Klf5 and Klf4, subsequently deciding the cellular fate. Furthermore, the inverse expression of Klf5 and Klf4 were also evaluated in kidneys from normal and UUO mice.

\section{Materials and Methods}

\section{Isolation of Primary mPTECs from Mice Kidneys}

Primary mPTECs from mice kidneys were harvested and cultured as previously described. ${ }^{12}$ All procedures were reviewed and approved through the Institute of Animal Care and Use Committee at the Medical College of National Cheng Kung University (Tainan, Taiwan).

\section{Microarray Database and Ingenuity Pathway Analysis}

RNA obtained from freshly isolated mPTECs and mPTECs cultured on culture dishes for 1 and 3 days were purified and quantified by OD260 $\mathrm{nm}$ by a ND-1000 spectrophotometer (Nanodrop Technology, Wilmington, DE) then qualitated by Bioanalyzer 2100 (Agilent Technologies, Santa Clara, CA) with RNA 6000 nano labchip kit. One microgram of total RNA was amplified by a low RNA input fluor linear amp kit (Agilent Technologies) and labeled with cyanin 3 (CyDye; PerkinElmer, Boston, MA) during the in vitro transcription process. Cyanin 3-labled complementary RNA $(1.65 \mu \mathrm{g})$ was fragmented to an average size of approximately 50 to 100 nucleotides by incubation with fragmentation buffer at $60^{\circ} \mathrm{C}$ for 30 minutes. Correspondingly fragmented labeled complementary RNA was

Table 1 Lists of the Top Six Up-Regulated and Down-Regulated Transcription Factors in Mouse Proximal Tubule Epithelial Cells During ex Vivo Culture

\begin{tabular}{|c|c|c|c|c|}
\hline Rank & Gene & Full name* & D1/D0 (fold) & D3/D0 (fold) \\
\hline \multicolumn{5}{|c|}{ Up-regulated } \\
\hline 1 & Klf5 & Kruppel-like factor 5 (NM_009769) & 25.2 & 28.3 \\
\hline 3 & Atf5 & Activating transcription factor 5 (NM_030693) & 15.2 & 5.5 \\
\hline 4 & $Y b \times 3$ (Csda) & Cold shock domain protein A (NM_011733) & 7.7 & 10.5 \\
\hline 5 & $E 2 f 3$ & E2F transcription factor 3 (NM_010093) & 6.3 & 4.6 \\
\hline 1 & Klf2 & Kruppel-like factor 2 (NM_008452) & -8.6 & -4.2 \\
\hline 2 & Atf3 & Activating transcription factor 3 (NM_007498) & -8.4 & -4.6 \\
\hline 3 & Sp5 & Trans-acting transcription factor 5 (NM_022435) & -6.9 & -4.5 \\
\hline 4 & Pitx2 & Paired-like homeodomain transcription factor 2 (NM_001042502) & -6.5 & -8.4 \\
\hline 5 & Klf15 & Kruppel-like factor 15 (NM_023184) & -5.4 & -16.9 \\
\hline
\end{tabular}

*Acquired from the NCBI Nucleotide Database (http://www.ncbi.nlm.nih.gov/nuccore).

$D$, day. 
then pooled and hybridized to oligo microarray (Agilent Technologies) at $60^{\circ} \mathrm{C}$ for 17 hours. After washing and drying by nitrogen gun blowing, microarrays were scanned with an Agilent microarray scanner at $535 \mathrm{~nm}$ for cyanin 3. Scanned images are analyzed by Feature extraction 10.5 software (Agilent Technologies), an image analysis and normalization software used to quantify signal and background intensity for each feature. The data discussed in this publication was deposited in National Center for Biotechnology Information's Gene Expression Omnibus ${ }^{36}$ (http://www.ncbi.nlm.nih.gov/ geo; accession number GSE69217). Ingenuity pathway analysis version 8.7 (Ingenuity Systems, Inc., Redwood City, CA) software was used for functional network analysis of the microarray result; Table 1 summarizes the results.

\section{UUO and 5/6 Nx in Mice}

All procedures were reviewed and approved by the Institute of Animal Care and Use Committee at the Medical College of National Cheng Kung University, Taiwan. UUO was performed in 1-month-old male C57BL/6 mice with an established procedure, as previously described. ${ }^{37,38}$ In mice subjected to UUO, the contralateral unligated kidney was used as a control organ. After UUO surgery, mice were sacrificed at various time points, and their kidneys were removed. Paraffin-fixed tissues were used for immunohistochemistry (IHC). Lysates from the whole kidney (including cortex and medulla) or cortex only were used for Western blot analysis. For in vivo lysyl oxidase inhibition, $\beta$-aminopropionitrile (BAPN; $200 \mathrm{mg} / \mathrm{kg}$ body weight; Sigma-Aldrich, St. Louis, MO) was injected via the i.p. route daily. In the control group, normal saline was used instead of BAPN. The injection was started 1 day before UUO surgery and persisted until the end of the experiment. In mice subjected to 5/6 nephrectomy (Nx), the left kidney was exposed, and the upper and lower poles were tied with a polyglycolic acid suture line, followed by right nephrectomy. Then, the peritoneum and skin were sutured. Seventeen weeks after 5/6 Nx surgery, mice were sacrificed, and their kidneys were removed for the IHC experiments.

\section{IHC}

IHC was performed, as previously described. ${ }^{39}$ Primary antibodies against Klf4, cyclin D1, proliferating cell nuclear antigen (Santa Cruz Biotechnology, Santa Cruz, CA), Klf5, YAP1 (Novus), phospho-extracellular signal-regulated kinase (p-ERK), and ERK (Cell Signaling, Boston, MA) were used for IHC detection. Part of the IHC experiments were performed by double staining polymer detection systems (BioTnA, Taiwan).

\section{Cell Lines}

$293 \mathrm{~T}$ (human embryonic kidney cell line), LLC-PK1 (porcine proximal tubule cell line), TCMK-1 (mice proximal tubule cell line), MDCK (dog distal tubule cell line),
M1 (mouse collecting duct cell line), and NRK49F (rat renal fibroblasts) cells were maintained in Dulbecco's modified Eagle's medium, supplemented with 5\% fetal bovine serum, $100 \mathrm{IU} / \mathrm{mL}$ penicillin, and $100 \mu \mathrm{g} / \mathrm{mL}$ streptomycin under $5 \% \mathrm{CO}_{2}$ at $37^{\circ} \mathrm{C}$.

\section{Preparation of Matrix}

Matrices composed of Matrigel (MG; BD Biosciences PharMingen, San Jose, CA) were prepared as previously described. ${ }^{12}$ The Young's moduli of these matrices were measured with atomic force microscopy (AFM). Briefly, the Young's modulus of the MG is approximately $66.0 \pm 0.3 \mathrm{~Pa}$, and both the dish and MG-coated dish are approximately giga $\mathrm{Pa}$.

\section{Western Blot Analysis}

Western blot analysis was performed as previously described. ${ }^{40}$ The cell lysates were harvested, resolved on SDSPAGE, and then electrophoretically blotted onto nitrocellular paper. The primary antibodies used in this study are listed as follows: Klf4, cyclin D1, glyceraldehyde-3-phosphate dehydrogenase (Santa Cruz Biotechnology), Klf5 (Millipore, Temecula, CA), $\alpha$-smooth muscle actin (Sigma-Aldrich), $\beta 1$ integrin (BD Biosciences PharMingen), p-ERK, and ERK (Cell Signaling).

\section{RT-PCR}

Total RNA was extracted with TRIzol reagent (InvitrogenMolecular Probes, Carlsbad, CA) according to the manufacturer's instructions. RNA quality was verified and reverse transcribed by Moloney murine leukemia virus reverse transcriptase (Promega, Madison, WI). PCR was performed with specific primer sets at $94^{\circ} \mathrm{C}$ for 5 minutes, followed by $27 \mathrm{cy}$ cles at $94^{\circ} \mathrm{C}$ for 30 seconds, $60^{\circ} \mathrm{C}$ for 30 seconds, and $72^{\circ} \mathrm{C}$ for 30 seconds, and a final step at $72^{\circ} \mathrm{C}$ for 7 minutes. The cDNA was then used as a template for PCR with the use of primers specific for mouse cyclin D1 (forward, 5'-CACACGGACTACAGGGGAGT-3'; reverse, 5'-CAAGGGAATGGTCTCCTTCA-3'); mouse Klf5 (forward, 5'-AGACGGCAGTAATGGACACC-3'; reverse, 5'-GATGTTGGCCTTCACGTACT- ${ }^{\prime}$ ); mouse Klf4 (forward, 5'-TAGCCTAAATGATGGTGCTTGGTG-3'; reverse, 5'-TGTTCTGCTTAAGGCATACTTGGG-3 ${ }^{\prime}$ ), and mouse glyceraldehyde-3-phosphate dehydrogenase (forward, 5'-ACGGCACAGTCAAGGCTGAG-3'; reverse, 5'-GGAGGCCATGTAGACCATGAGG- $\left.3^{\prime}\right)$. The PCR products were separated on a $1.2 \%$ agarose gel that contained ethidium bromide and was visualized under a UV transilluminator.

\section{Immunofluorescence Staining}

Immunofluorescence staining was performed as previously described. ${ }^{41}$ The primary antibodies used in this study are listed as follows: cyclin D1, Klf4 (Santa Cruz Biotechnology), 
Klf5, and YAP1 (Novus, Littleton, CO). After washing with phosphate-buffered saline, the cells were incubated with the secondary antibody for anti-mouse or rabbit IgG conjugated with Alexa 488 (Invitrogen-Molecular Probes) and/or phalloidin-tetramethylrhodamine isothiocyanate (Sigma-Aldrich) and $10 \mu \mathrm{g} / \mathrm{mL}$ Hoechst 33258 for 1 hour. The imaging was performed from sequential $\mathrm{z}$-series scans with a confocal microscope (FV-1000; Olympus, Tokyo, Japan). cyclin D1, Klf5, and YAP1 in the apical, middle, and basal regions of cells were recolored green, red, and blue, respectively. The Max XY projection images were reconstructed from a stack of recolored confocal images by ImageJ software version 1.410 (NIH, Bethesda, MD; http://imagej.nih.gov/ij).

\section{Cellular Fractionation}

Nuclear and cytoplasmic fractions were obtained with the REAP (Rapid, Efficient and Practical) method. ${ }^{42}$ Briefly, cells grown on dishes were washed with phosphate-buffered saline and then scraped from dishes. After quickly spinning, the pellets were resuspended in $900 \mu \mathrm{L}$ ice-cold $0.1 \%$ NP-40 (Calbiochem, La Jolla, CA) in phosphate-buffered saline and titrated to mechanically disrupt the cytoplasmic membranes. Lysate $(300 \mu \mathrm{L})$ was divided into aliquots as the whole cell lysate. After the second centrifugation, 300 $\mu \mathrm{L}$ of the supernatant fluid was divided into aliquots as the cytoplasmic fraction. The resulting pellet was washed with 1 $\mathrm{mL}$ ice-cold $0.1 \%$ NP-40 and centrifuged again. The pellet was then resuspended with $180 \mu \mathrm{L} 1 \times$ Laemmli sample buffer and designated the nuclear fraction. One hundred microliter of $4 \times$ Laemmli sample buffer was added to the whole cell and cytoplasmic fractions. Each fraction was sonicated with microprobes and then boiled for 1 minute. Finally, the whole cell, cytoplasmic, and nuclear fractions were examined by Western blot analysis.

\section{Assessment of Tissue/Cell Mechanical Properties by AFM}

For measurements of mechanical properties of tissue/cell, JPK NanoWizard II AFM with BioCell (JPK Instruments, Berlin, Germany) was equipped and manipulated as previously described. ${ }^{43}$ Fresh kidney tissue samples were sliced at a thickness of $100 \mu \mathrm{m}$ with a microtome. Tissue slices were glued to a glass coverslip with a small drop of nail polish, and only the intact side of the cortex was immediately subjected to AFM measurements. Tipless cantilevers (Arrow-TL1-50;

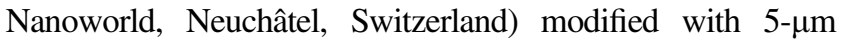
diameter polystyrene bead were used to measure tissue and cells. The spring constants of all cantilevers were calibrated via the thermal noise method in liquid before each measurement and valued $0.03 \mathrm{~N} / \mathrm{m}$. The indenting force was set at $1 \mathrm{nN}$. Force-distance curves were collected and calculated with JPK package software version 4.6.62 (JPK Instruments), which was based on the Hertz model.

\section{Establish mCherry-Klf4 Expression Clones and Plasmid} Construction

For transient transfection, 293T cells, LLC-PK1 cells, and mPTECs were plated on culture dishes for 24 hours before transfection with Lipofectamine 3000 plus reagent according to the manufacturer's instructions (Life Technologies, Inc., Carlsbad, CA). The plasmids of p-mCherry and pLMmCherry-Klf4 were purchased from Addgene Inc. (Cambridge, MA). After transfection, images of the transfected cells were taken to measure the cell spreading area, and then lyzed for RT-PCR. To further enrich the mCherry-positive cells in $293 \mathrm{~T}$ cells, cells were sorted by flow cytometry. For immunostaining in transfected mPTECs, the medium was replaced for another 48 hours of culture after transfection. The cells were then fixed and costained with cyclin D1 and Hoechst 33258.

\section{Establish Lentivirus-Delivery shRNA of Klf5}

To knockdown Klf5 in mPTECs cells, 21-mer shRNA against mouse Klf5 expressed in pLKO.1 vector was purchased from National RNAi Core Facility (Taipei, Taiwan). The sequence for shKlf5 is $5^{\prime}$-TCCGATAATTTCAGAGCATAA-3'.

\section{Evaluation of Cell Proliferation with Click-iT Edu Kits}

Cell proliferation was evaluated by Click-iT EdU (5-ethynyl2'-deoxyuridine) Alexa Fluor 488 Imaging Kit (InvitrogenMolecular Probes) as previously described. ${ }^{12}$ Briefly, mPTECs were cultured on the indicated conditions for 3 days and incubated with EdU for 7.5 hours before analysis. For some experiments, cells were incubated with primary rabbit antibody against Klf5 (Novus) at $4^{\circ} \mathrm{C}$ overnight. After extensively rinsing with phosphate-buffered saline, the cells were incubated with the secondary antibody for anti-rabbit IgG conjugated with Alexa 594 (Invitrogen-Molecular Probes) and $10 \mu \mathrm{g} / \mathrm{mL}$ Hoechst 33258 for 1 hour. The immunocomplexes were visualized with confocal microscopy (FV-1000; Olympus, Tokyo, Japan).

\section{Statistical Analysis}

All data were expressed as means \pm SEM of at least three independent experiments. One-way analysis of variance was used to compare differences when a group contained more than three members. $P<0.05$, as calculated by GraphPad Prism version 3.0 (GraphPad Software, San Diego, CA), was considered statistically significant.

\section{Results}

Proliferative Potential of mPTECs Positively Correlates with Mechanical Cues from Matrix Stiffness

When freshly isolated mPTECs were plated on culture dishes, the three-dimensional tubule first shrank and 
aggregated. Cells in the basal layer of the aggregated mass then started to spread (Figure 1A). The expression of cyclin D1 mRNA, a proliferation marker, was elevated at 24 hours and persisted until 120 hours (Figure 1B). To clarify the molecules involved in cell proliferation, we extracted the possible TFs relevant to regulate cyclin D1 expression from the oligo-microarray profiling of MPTECS on culture dishes for 1 day. Among all of the TFs, Klf5 was the most dramatically up-regulated TF. Meanwhile, Klf4 exerted the opposite effect of Klf5 and was dramatically downregulated (Table 1). The RT-PCR results confirmed that Klf5 increased and Klf4 decreased significantly at 2 hours, and this condition was maintained during culture (Figure 1B). Western blot analysis found that both cyclin D1 and Klf5 markedly increased, whereas Klf4 decreased at 72 hours (Figure 1, C and D).

To visualize the spatial distribution and the correlation between cyclin D1 and Klf5 in mPTECs during ex vivo culture, confocal immunofluorescence staining was performed. The results found that the freshly isolated mPTECs displayed low intensity of cyclin D1 and Klf5. In addition, the faint Klf5 was mainly located in the cytosol. The intensity and nuclear translocation of cyclin D1 and Klf5 significantly increased with time (Figure 2, A and B). Cellular fractionation results detected the mature Klf5 (mol. wt., $52 \mathrm{kDa}$ ) on day 1 after culture, which increased with time (Figure 2C). The XZ-sections of these images revealed that cells with nuclear cyclin D1 and Klf5 were mainly located at the basal region of the MPTEC aggregate (Figure 2D). To better evaluate the distribution of nuclear cyclin D1 and Klf5-positive cells in the MPTEC aggregate, we generated Max XY projection images from a stack of sequential z-series scan confocal microscope images. The stain in the apical, middle, and basal regions of the mPTEC aggregate were recolored blue, red, and green, respectively (Figure 2E). The quantification results revealed that most nuclear cyclin D1- and Klf5-positive cells, shown in green, were located in the basal region of the MPTEC aggregate (Figure 2F).

In the fibrotic kidneys induced by UUO or 5/6 Nx, Klf5 increased in proliferative tubular cells that were located in both the cortex and medulla region (Supplemental Figure S1, A and B). The double staining of Klf5 and aquaporin 1 (a marker of PTs) confirmed that Klf5 was expressed in PTs of fibrotic kidneys (Supplemental Figure S1, C and D). However, Klf5 was not expressed in CD31-positive endothelial cells and fibroblasts (Supplemental Figure S1, E and F, respectively). In addition to the result of the inflammatory effect in collecting duct cells, ${ }^{32}$ we speculate whether Klf5 has the proliferation effect in PTs. Our colleagues found that tissue stiffness of obstructed kidney was significantly increased on day 7 after UUO (Y.C. Yeh, unpublished data). Considering the importance of matrix stiffness in the regulation of cell proliferation, we determined whether and how the mechanical stimulus regulates Klf5 expression and cell proliferation in PTs with the use of in vivo and in vitro experimental model systems.

Cells in the basal region of the MPTEC aggregate displayed the highest proliferative potential as confirmed by high incorporated EdU (Supplemental Figure S2, A and B) and the well-organized stress fiber (Supplemental Figure S2C). AFM indentation results found that the Young's modulus of mPTECs on day 3 after culturing on a plastic dish was significantly higher than those of freshly isolated mPTECs or cortex tissue (Supplemental Figure S2,
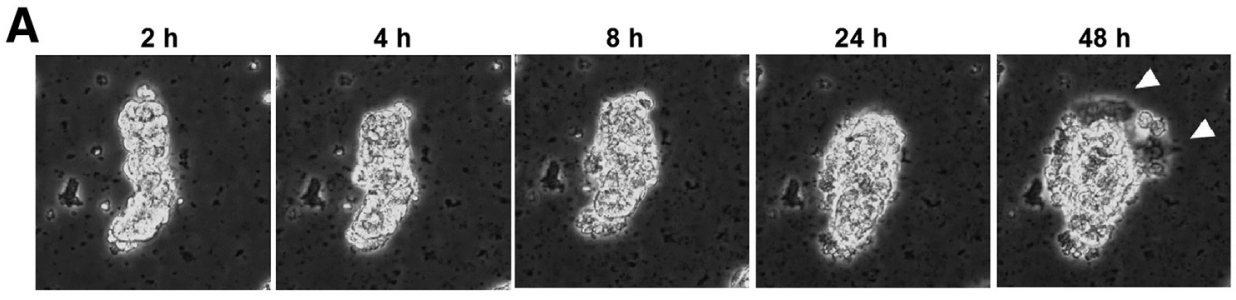

$72 \mathrm{~h}$
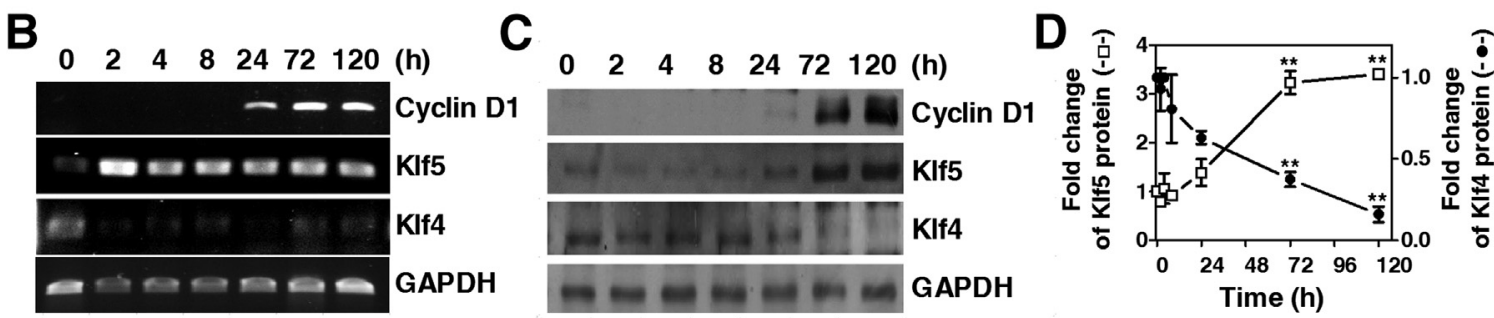

Figure 1 Inverse gene expression patterns for Klf5 and Klf4 in ex vivo culture of mPTECs. Primary mPTECs were isolated and cultured on culture dishes. A: Time-lapse phase contrast microscopy images show that mPTECs begin to spread in the lowest layer of the tubular aggregate (arrowheads). B: Representative RT-PCR results of mPTECs at the indicated times. The mRNA expression of cyclin D1, Klf5, and Klf4 were analyzed. GAPDH was used as an internal control. C: Representative Western blot analysis results of mPTECs at the indicated times. The protein levels of cyclin D1, Klf5, and Klf4 were analyzed. D: Quantification results of Klf5 and Klf4 are from C. GAPDH was used as an internal control. GAPDH-normalized data in each condition were compared with those of cells on day $0 .{ }^{*} P<0.01$. Scale bar $=40 \mu \mathrm{m}$. GAPDH, glyceraldehyde-3-phosphate dehydrogenase; Klf, Krüppel-like factor; mPTEC, mouse proximal tubule epithelial cell. 


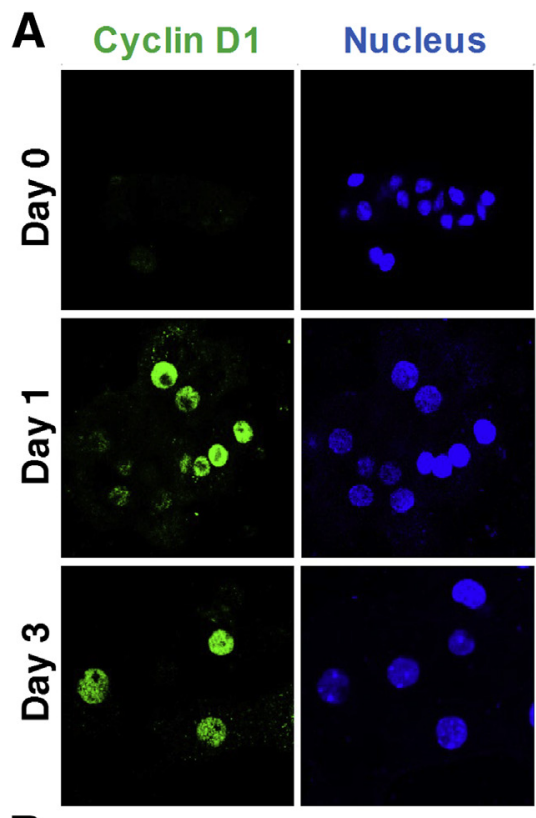

B

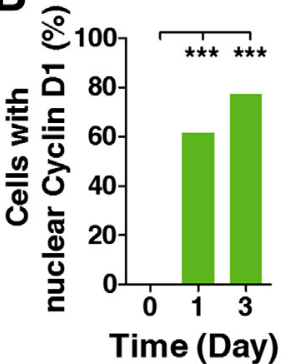

D
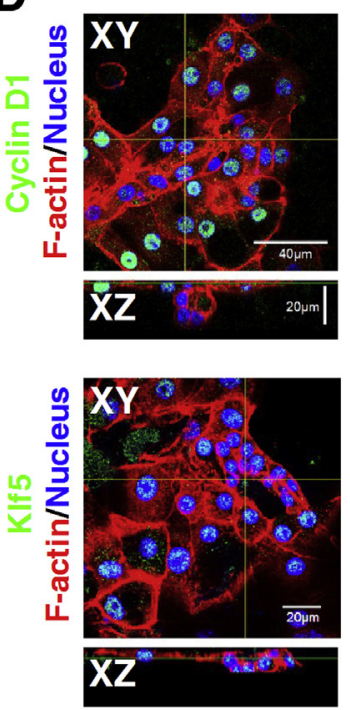
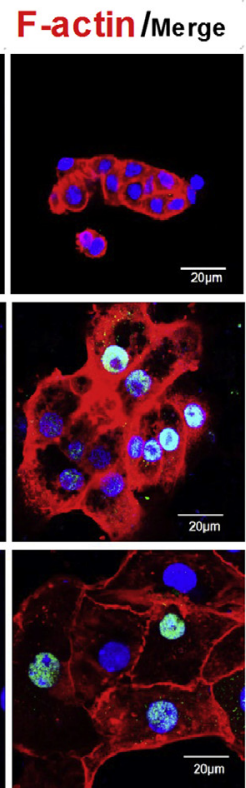

C

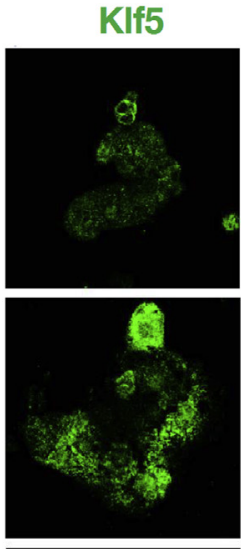

Nucleus

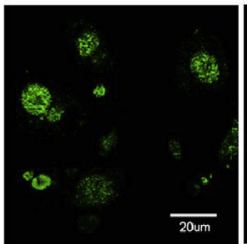

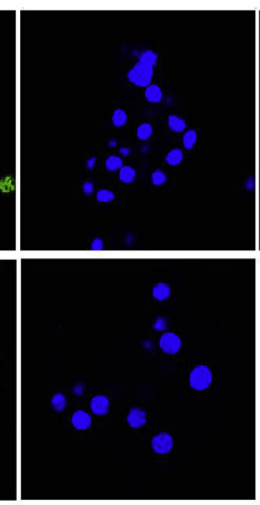

F-actin/Merge
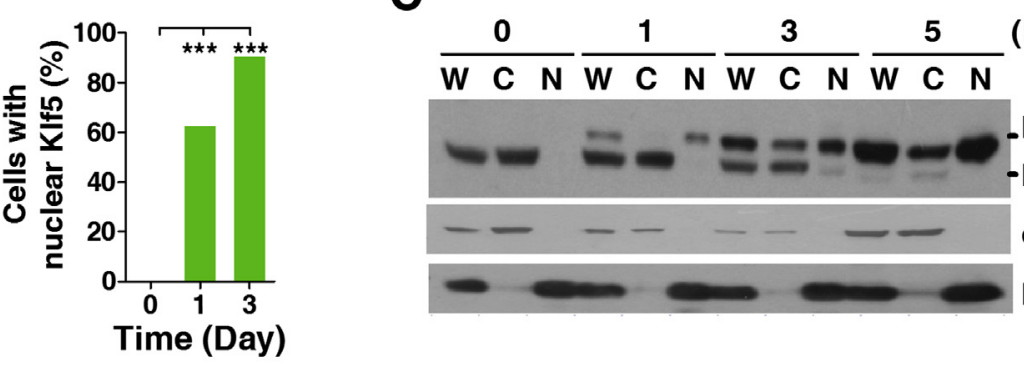

(Day)
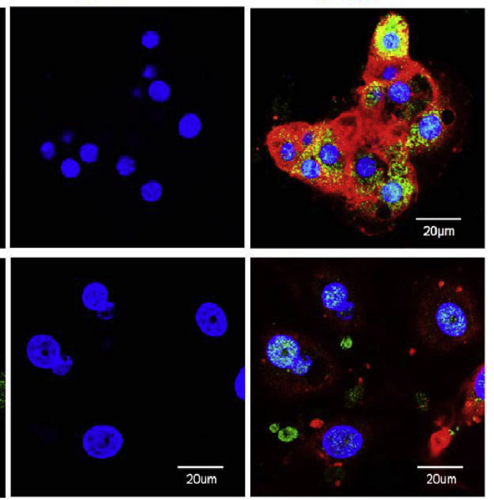

Klf5

Degrad. Klf5

a-tubulin

Histone H3
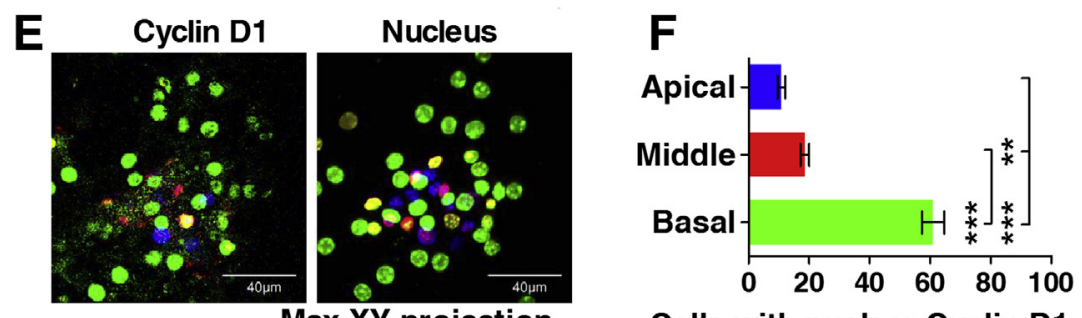

Cells with nuclear Cyclin D1 (\% of total cells)
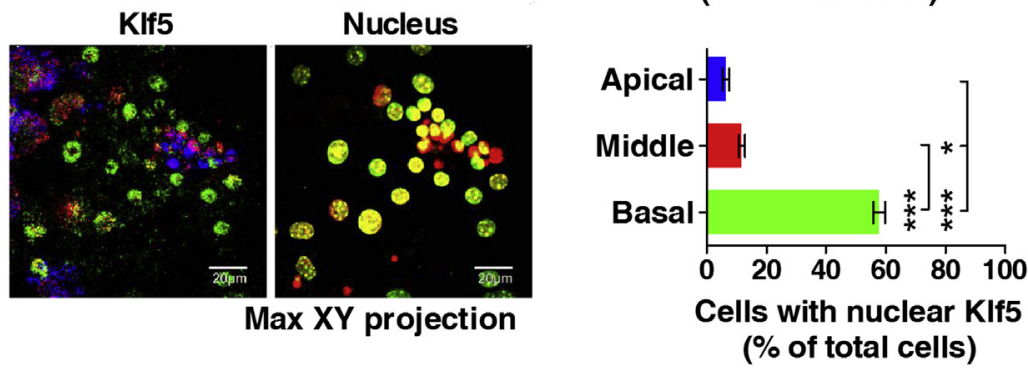

Figure 2 The spatial distribution of nuclear cyclin D1 and Klf5 in ex vivo culture of (mPTECs). Primary mPTECs were isolated and cultured on culture dishes. A: Confocal immunofluorescence images of mPTECs at the indicated times. Cells were stained for cyclin D1 (green, left panel) and Klf5 (green, right panel) with the costaining of nucleus (blue) and F-actin (red). B: Frequency of nuclear cyclin D1- and Klf5-positive cells in mPTECs at the indicated times from A. C: Representative subcellular fractionation results for mPTECs at the indicated times. Nuclear and cytoplasmic proteins were separated by the REAP method (Materials and Methods). The protein levels of Klf5 and Degrad Klf5 were analyzed. $\alpha$-Tubulin and histone H3 served as cytoplasmic and nuclear markers, respectively. D: Confocal immunofluorescence images of cyclin D1 (green, upper panel) or Klf5 (green, lower panel) with the costaining of F-actin (red), and nucleus (blue) in XY and XZ sections of mPTECs at day 3. E: Representative Max XY projection images of mPTECs reconstructed from D. Cyclin D1 or Klf5 with nucleus in the apical, middle, and basal regions of the mPTEC aggregate were recolored into blue, red, and green, respectively. F: The distribution of nuclear cyclin D1- or Klf5-positive cells in the apical, middle, and basal regions of the mPTEC aggregate were evaluated from E. ${ }^{*} P<0.05,{ }^{*} P<0.01$, and ${ }^{*} * P<0.001$. C, cytoplasmic fraction; Degrad, degraded; Klf5, Krüppel-like factor 5; mPTEC, mouse proximal tubule epithelial cell; N, nuclear fraction; REAP, Rapid, Efficient and Practical; W, whole cell lysate. 
D and E). Moreover, the peripheral mPTECs displayed more stress fiber staining and higher Young's modulus than the central aggregated cells (Figure 3A and Supplemental Figure S2, C and E). When cultured on soft MG, mPTECs retained cortical actin and mechanical properties similar to fresh mPTECs. Thus, we were curious about whether alterations in matrix stiffness influenced the inverse expression of Klf4 and Klf5, which was linked to the regulation of cell proliferation. RT-PCR results found that soft MG stunted the expression of K1f5 and cyclin D1 and preserved
A

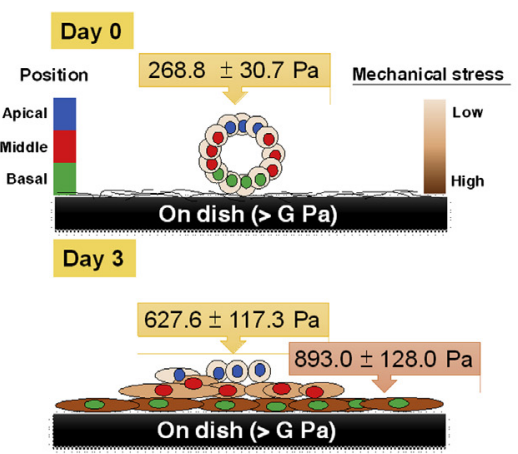

B

$$
\text { Day } 3
$$

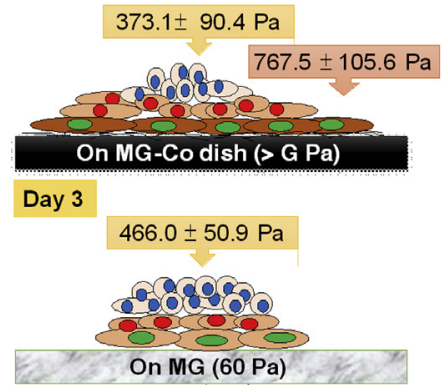

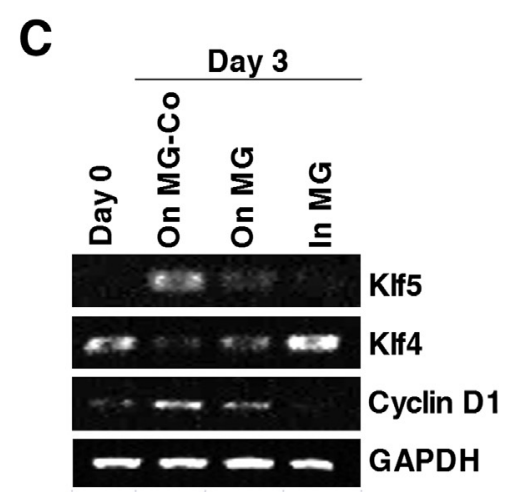

D
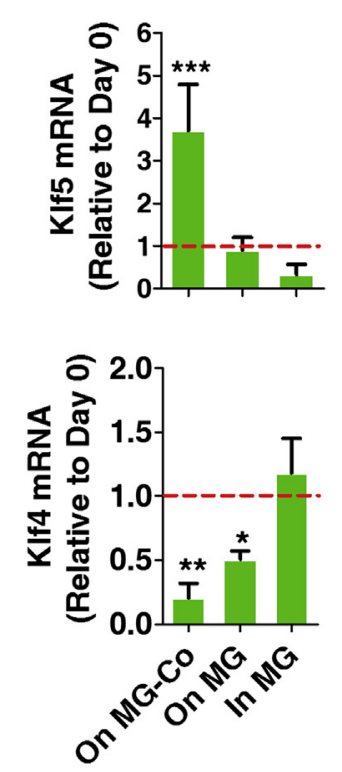

G
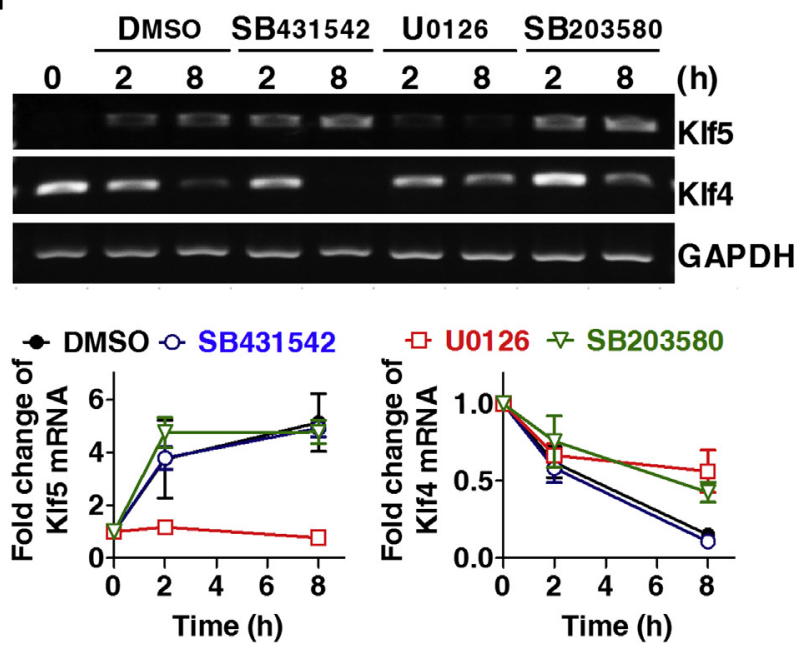

E

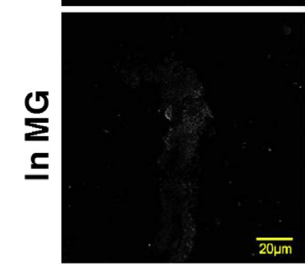

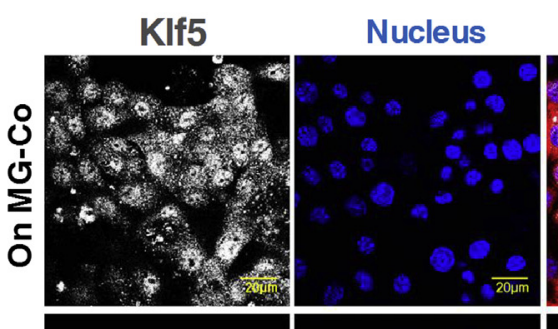
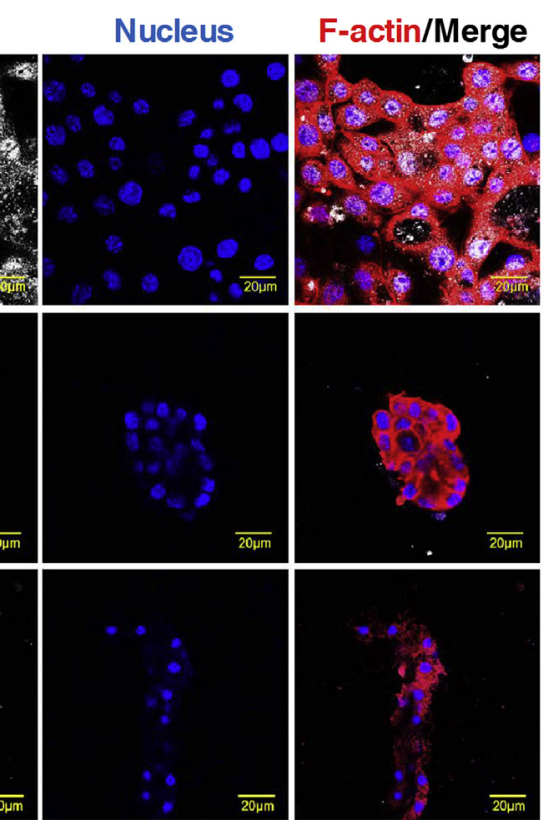

H

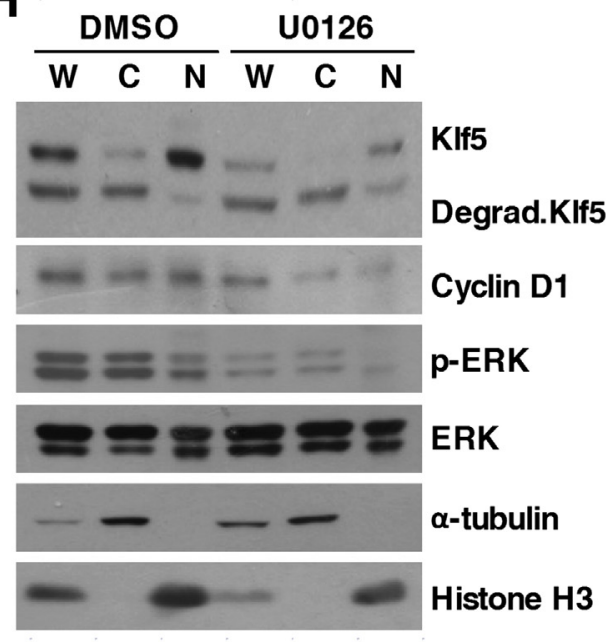


Klf4, suggesting growth arrest in mPTECs (Figure 3, C and D). Confocal immunofluorescence images further confirmed that soft matrix successfully repressed Klf5 intensity and nuclear translocation (Figure 3, E and F). Taken together, we propose that the cells in the basal region of the mPTECs aggregating directly adjacent to the stiff matrix receive the strongest mechanical cues and respond by the augmentation of cell spreading and proliferation. In contrast, the cells in the middle and apical regions of mPTECs receive less or no mechanical cues from the matrix stiffness; hence, they remain quiescent. Thus, the spatial distribution of cell proliferation and nuclear Klf5 might positively correlate with the mechanical cues from matrix stiffness.

To clarify the detailed mechanisms underlying stiff matrix-induced inverse expression of Klf5/Klf4, mPTECs were treated with several inhibitors, including $10 \mu \mathrm{mol} / \mathrm{L}$ SB431542 (transformation growth factor- $\beta 1$ receptor inhibitor), $20 \mu \mathrm{mol} / \mathrm{L}$ U0126 (mitogen-activated protein ERK kinase/ERK inhibitor), and $20 \mu \mathrm{mol} / \mathrm{L}$ SB203580 (p38 mitogen-activated protein kinase inhibitor). RT-PCR results found that stiff matrix-induced gain of Klf5 was completely blocked by U0126, and the loss of Klf4 was partially suppressed by either U0126 or SB203580 (Figure 3G). Further, inhibition of ERK activity not only suppressed the protein amount but also interfered with the nuclear translocation of Klf5 (Figure $3 \mathrm{H}$ ). Taken together, these data suggest that ERK activity plays a critical role in stiff matrix-induced Klf5 up-regulation and Klf4 downregulation. In addition, p38 mitogen-activated protein kinase activity also partially contributed to regulating Klf4 expression.

\section{Gain of Klf5 and Loss of Klf4 Contribute to Stiff Matrix-Induced Cell Spreading and Proliferation}

Considering the positive correlation of inversely expressed Klf5/4 with cell proliferation and cyclin D1 expression, we evaluated the functional divergence between Klf5 and Klf4 in stiff matrix-induced cell proliferation. First, mPTECs were subjected to lentiviral infection with Klf5-specific
shRNA to examine the role of Klf5. When cultured on stiff matrix, phase-contrast images found that not only cell proliferation but also cell spreading was restricted by knockdown of Klf5 (Figure 4A). RT-PCR results confirmed that the Klf5 mRNA was significantly attenuated in mPTECs transfected with shKlf5 compared with cells transfected with nonspecific shRNA at day 5 . Knockdown of Klf5 by shKlf5 also abolished stiff matrix-up-regulated cyclin D1 mRNA (Figure 4B). This phenomenon was further confirmed by the treatment of Am80 (a Klf5 inhibitor) ${ }^{44}$ at a dose of $10 \mu \mathrm{mol} / \mathrm{L}$ (Figure 4C). Am80 treatment indeed suppressed cell proliferation as confirmed by EdU assay (Supplemental Figure S3, A and B). shKlf5 stunted stiff matrix-induced Klf4 mRNA down-regulation, implying a regulatory loop to regulate the inverse expression of Klf4 and Klf5 (Figure 4B). To examine the role of Klf4 loss, cells were transfected with p-mCherry or with pLM-mCherry-Klf4. Both the RT-PCR and immunofluorescence image results confirmed the forced expression of Klf4 in pLM-mCherry-Klf4-transfected 293T cells (Supplemental Figure S4, A and B). After sorting, mCherry- and mCherry-Klf4-positive cells were separately enriched and cultured on culture dishes. mCherry-expressing 293T cells spread and proliferated to form small colonies after 3 days, whereas mCherry-Klf4-transfected 293T cells remained rounded and in a state of arrested growth (Supplemental Figure S4C). To further confirm the effect of Klf4 on cell spreading, the cell area of the mCherry- or mCherry-Klf4-transfected cells were measured. Forced expression of Klf4 significantly decreased cell area in mPTECs, 293T cells, and LLC-PK1 cells, suggesting that Klf4 overexpression-restricted cell spreading was a general phenomenon (Figure 4D and Supplemental Figure S4, D and E). Confocal immunofluorescence images further found that forced expression of Klf4 in mPTECs suppressed nuclear cyclin D1 and EdU intensity compared with Mock control or mCherrypositive cells (Figure 4, E and F, and Supplemental Figure S3, C and D). Taken together, these data confirm that matrix stiffness-regulated Klf5 and Klf4 play work in opposition as regulators: Klf5 promotes cell spreading and cyclin D1 expression, whereas Klf4 suppresses them.

\footnotetext{
Figure 3 Stiff matrix triggers Klf5 up-regulation and Klf4 down-regulation via ERK activation. Proposed diagram shows the spatial distribution of mechanical properties in a freshly isolated or cultured mPTECs on dishes (A), and cultured mPTECs on MG-Co dishes or MG for 3 days (B). The Young's modulus of the periphery or central aggregated mPTECs were obtained from Supplemental Figure S1, D and E. Cells in the basal layer (green) of mPTEC aggregate received the highest mechanical stress and display the best spreading ability compared with those in the middle (red) and apical layers (blue) of the mPTEC aggregate. C: Representative RT-PCR results of mPTECs cultured on MG-Co, on MG, or in MG for 3 days. The mRNA expressions of Klf5, Klf4, and cyclin D1 were analyzed. GAPDH was used as an internal control. D: Quantification results of Klf5 and Klf4 mRNA from C. GAPDH-normalized data in each condition were compared with those of cells on day 0 (dashed line). E: Confocal immunofluorescence images of mPTECs on different culture matrices. Cells were stained for Klf5 (gray), nucleus (blue), and F-actin (red). F: Frequency of nuclear Klf5-positive cells in mPTECs at the indicated substrates from E. G: Representative RT-PCR results for the effect of DMSO (control), $10 \mu \mathrm{mol} / \mathrm{L}$ SB431542 (transformation growth factor- $\beta 1$ receptor inhibitor), $20 \mu \mathrm{mol} / \mathrm{L}$ U0126 (mitogen-activated protein ERK kinase inhibitor), and $10 \mu \mathrm{mol} / \mathrm{L} \mathrm{SB203580} \mathrm{(p38} \mathrm{mitogen-activated} \mathrm{protein} \mathrm{kinase} \mathrm{inhibitor)} \mathrm{on} \mathrm{the} \mathrm{mRNA} \mathrm{expression} \mathrm{of} \mathrm{Klf5} \mathrm{and} \mathrm{Klf4} \mathrm{in} \mathrm{mPTECs} \mathrm{cultured} \mathrm{on}$ cultured dishes at the indicated times. GAPDH was used as an internal control. Quantification results of Klf5 and Klf4 mRNA. GAPDH-normalized data in each condition were compared with those of cells on day 0 . H: Representative subcellular fractionation results for the effect of U0126. The protein levels of Klf5, Degrad. Klf5, cyclin D1, p-ERK, and ERK were analyzed. $\alpha$-Tubulin and histone H3 served as cytoplasmic and nuclear markers, respectively. ${ }^{*} P<0.05$, ${ }^{* * P}<0.01$, and ${ }^{* *} P<0.001$. C, cytoplasmic fraction; Degrad, degraded; DMS0, dimethyl sulfoxide; ERK, extracellular signal-regulated kinase; GAPDH, glyceraldehyde-3-phosphate dehydrogenase; Klf, Krüppel-like factor; MG, Matrigel; MG-Co, Matrigel-coated dish; mPTEC, mouse proximal tubule epithelial cell; $\mathrm{N}$, nuclear fraction; W, whole cell lysate.
} 


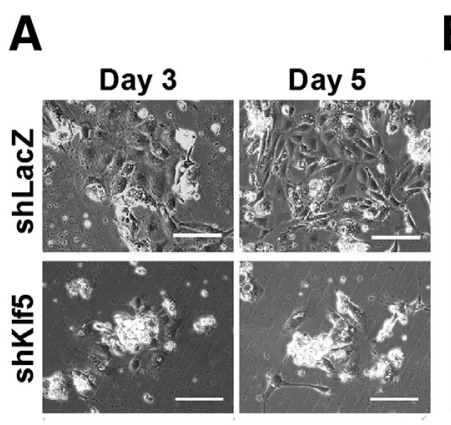

B

D

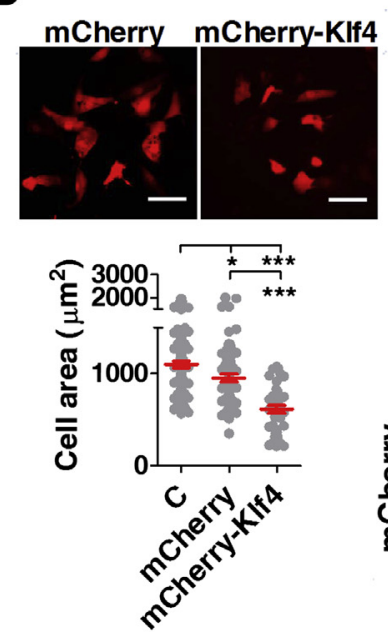

$\mathbf{E}$

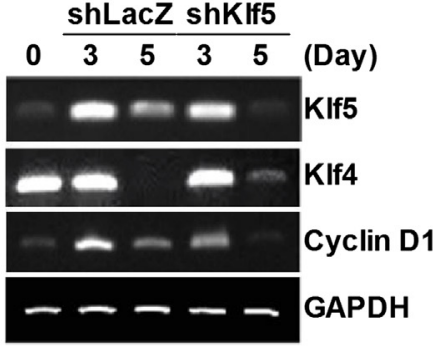

C

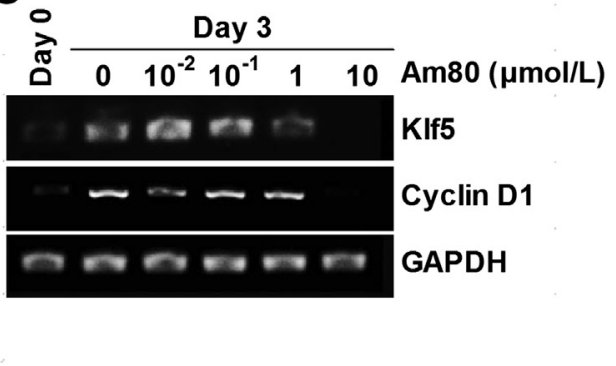

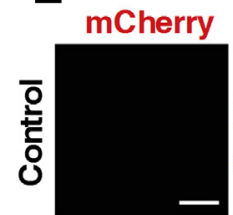
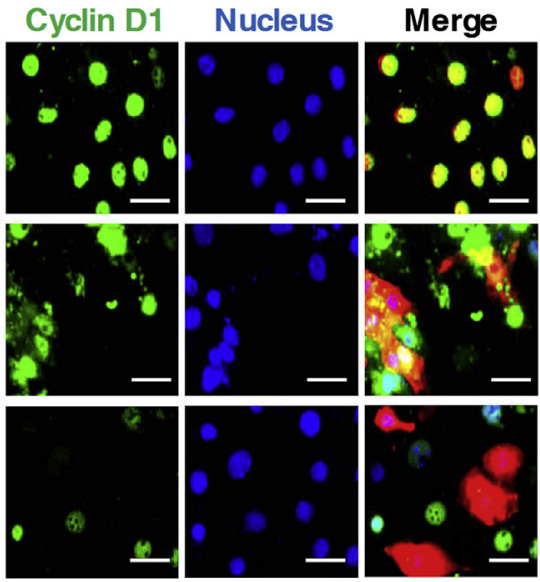

$\mathbf{F}$
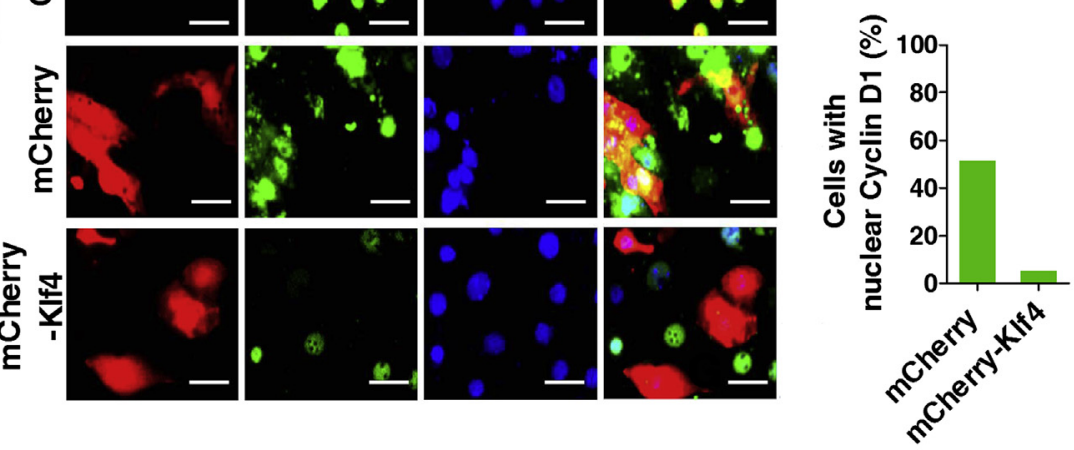

Figure 4 The suppression of Klf5 by lentivirus-mediated shRNA or Am80 or forced overexpression of Klf4 stunts cell spreading and cell growth. A: Phase contrast images of mPTECs transduced with shLacZ or shKlf5 for the indicated times. B: Representative RT-PCR results of mPTECs transduced with shLacZ or shKlf5 for the indicated time. mPTECs were cultured on culture dishes for 4 hours and then targeted to deplete Klf5 through lentiviral shRNA. The mRNA expressions of Klf5, Klf4, and cyclin D1 were analyzed. GAPDH was used as an internal control. C: Representative RT-PCR results of mPTECs treated with different doses of Am80 for 3 days. The mRNA expressions of Klf5 and cyclin D1 were analyzed. GAPDH was used as an internal control. D: Immunofluorescence images of mPTECs transfected with mCherry or mCherry-Klf4 for 3 days. The lower panel shows the cell areas of control (without transfection), mCherry-, or mCherry-Klf4-transfected mPTECs. E: Confocal immunofluorescence images of mCherry- or mCherry-Klf4-transfected mPTECs on day 3. Cells were stained for cyclin D1 (green) and nucleus (blue). F: Percentage of nuclear cyclin D1 within mCherry in mCherry control and mCherry-Klf4-transfected mPTECs were assessed from E. $n=44$, mCherry control mPTECs (F); $n=50$, mCherry-Klf4-transfected mPTECs (F). ${ }^{*} P<0.05,{ }^{* *} P<0.001$. Scale bars: $100 \mu \mathrm{m}(\mathbf{A}$ and D); $20 \mu \mathrm{m}$ (E). Am80, Klf5 inhibitor; C, control; GAPDH, glyceraldehyde3-phosphate dehydrogenase; Klf, Krüppel-like factor; mPTEC, mouse proximal tubule epithelial cell; shLacZ, nonspecific shRNA.

\section{Stiff Matrix-Activated YAP1 Is Relevant to the Up-Regulation of Klf5}

When cultured on stiff matrix, Klf5 mRNA was markedly elevated within 2 hours, whereas Klf5 protein was enhanced after 72 hours (Figure 2, A and B). In addition, the Western blot analysis results found that Klf5 existed in a degraded form (mol. wt. $=43 \mathrm{kDa}$ ) at day 0 and gradually developed into a mature form (mol. wt. $=52 \mathrm{kDa}$ ) over time (Figure 2C). Post-translational regulation might thus also be involved in the stiff matrix-induced Klf5 up-regulation. Klf5 is an unstable protein and is easily degraded by ubiquitinmediated proteolysis in both normal and transformed epithelial cells. ${ }^{45-47}$ Recently, studies reported that YAP1 stabilized Klf5 by directly binding in the nucleus and prevented its degradation by E3 ubiquitin ligase WWP $1 .{ }^{48}$ YAP1, a coactivator in the Hippo pathway, serves as a sensor and mediator of mechanical cues, including shear stress, stretch, and matrix stiffness. ${ }^{49}$ Confocal immunofluorescence images showed the enhancement of nuclear YAP1 during culture (Figure 5A). Moreover, the XZ-sections of images revealed that nuclear YAP1 was mainly stained in cells located at the basal region of the mPTEC aggregate at day 3 (Figure 5B). A Max XY projection of the spatially recolored images also confirmed this observation (Figure 5, C and D). The subcellular fractionation results further confirmed that the nuclear co-fractionation of YAP1 and mature Klf5 increased with time (Figures 2C and 5E). However, when cultured on soft MG, nuclear YAP1 was completely suppressed, compared with those on stiff MG-coated dish (Figure 5F). These data imply that YAP1 expression, like that of Klf5 and cyclin D1, is also mechanoresponsive. In addition, we applied U0126, which was reported to regulate Klf4/5 expression, and found that U0126 partially inhibited the nuclear distribution of YAP1 (Figure 5G). In summary, we suggest that stiff matrixactivated nuclear YAP1 may be critical for Klf5 maturation and nuclear translocation, which subsequently facilitates mPTEC proliferation. 

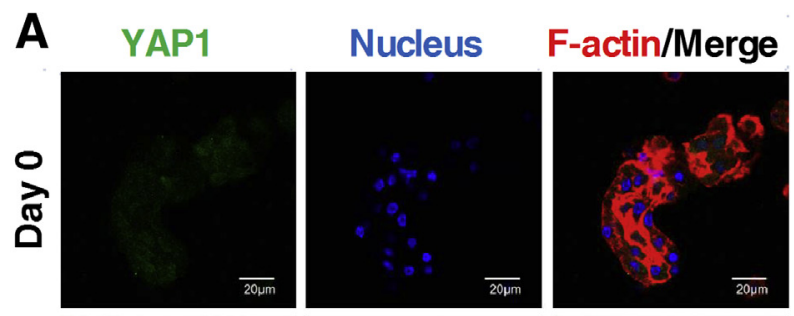

B
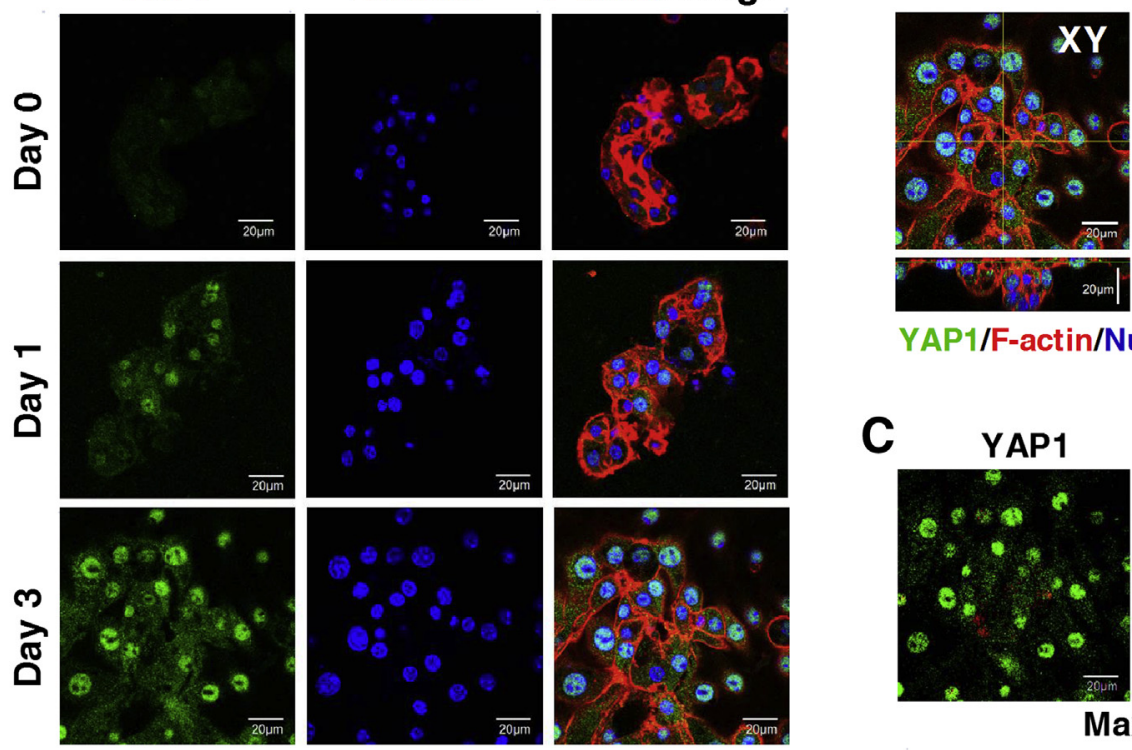

YAP1/F-actin/Nucleus

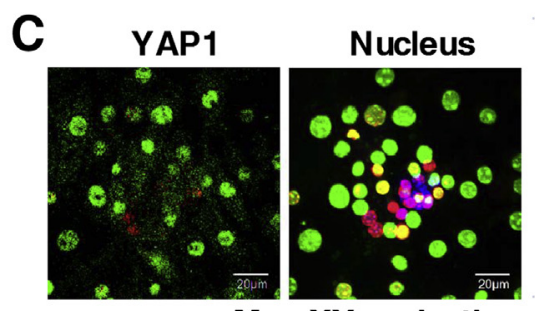

Max XY projection

D

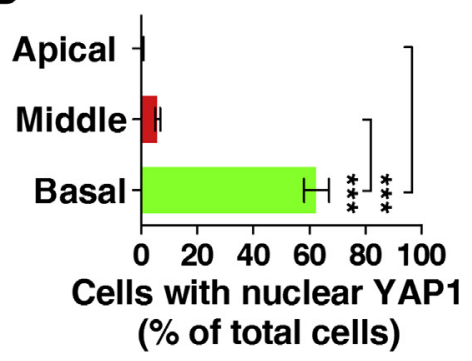

E
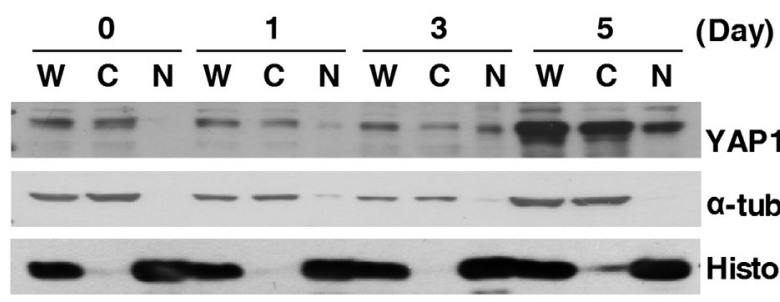

YAP1

a-tubulin

Histone H3

$\mathbf{F}$
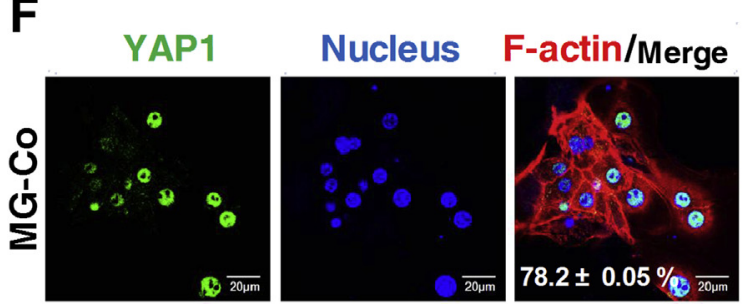

G
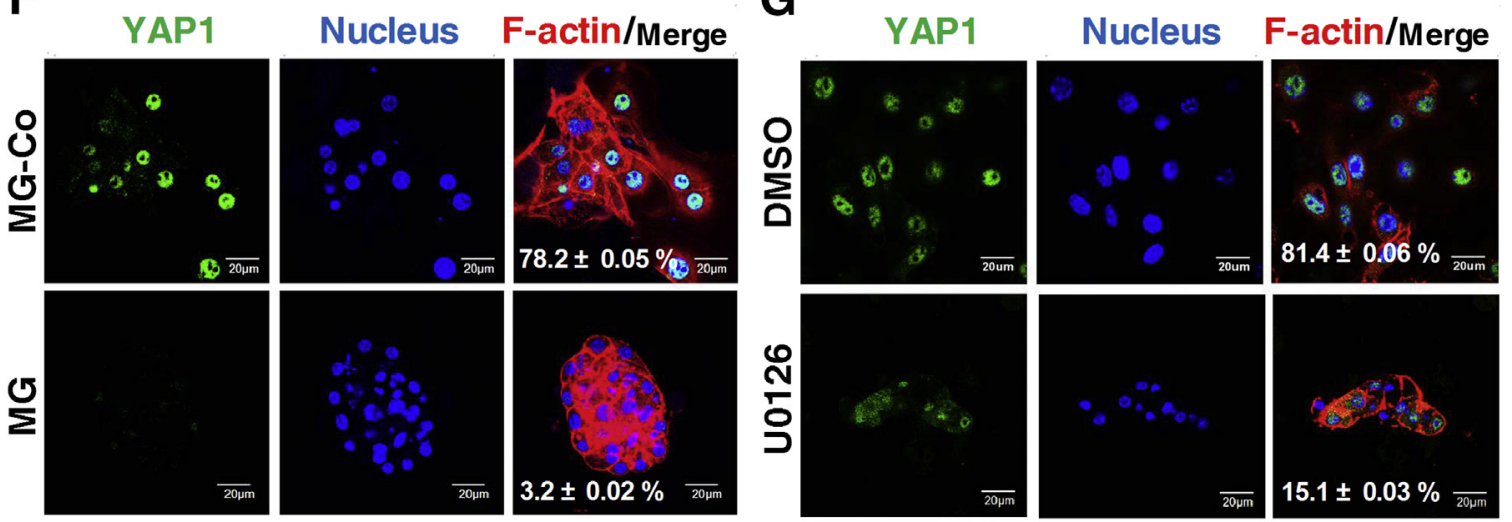

Figure 5 Stiff matrix facilitates the expression and nuclear translocation of YAP1, which is mediated via extracellular signal-regulated kinase activation. Primary mPTECs were cultured on culture dishes. A: Confocal immunofluorescence images of mPTECs at the indicated times. Cells were stained for YAP1 (green), nucleus (blue), and F-actin (red). B: Representative confocal immunofluorescence images of YAP1 (green), nucleus (blue), and F-actin (red) distributions in the XY and XZ sections of mPTECs at day 3. C: Representative Max XY projection images of mPTEC reconstructed from B. YAP1 (left) and nucleus (right) in the apical, middle, and basal regions of the mPTEC aggregate were recolored blue, red, and green, respectively. D: The distribution of nuclear YAP1-positive cells in the apical, middle, and basal regions of the MPTEC aggregate were evaluated from C. E: Representative subcellular fractionation results for MPTECs at the indicated times. The protein levels of YAP1 was analyzed. $\alpha$-Tubulin and histone $\mathrm{H} 3$ served as cytoplasmic and nuclear markers, respectively. F: Confocal immunofluorescence images of mPTECs cultured on MG-Co dishes or MG for 3 days. Cells were stained for YAP1 (green), nucleus (blue), and F-actin (red). The value shown in the merged image indicates the percentage of YAP1-positive cells in each condition. G: Confocal immunofluorescence images of mPTECs cultured on culture dishes treated with DMS0 (control) or $20 \mu \mathrm{mol} / \mathrm{L}$ U0126 for 3 days. Cells were stained for YAP1 (green), nucleus (blue), and F-actin (red). The value shown in the merge image indicates the percentage of YAP1-positive cells in each condition. ${ }^{* * *} P<0.001$. C, cytoplasmic fraction; DMSO, dimethyl sulfoxide; MG, Matrigel; MG-Co, Matrigel coated dish; mPTEC, mouse proximal tubule epithelial cell; N, nuclear fraction; W, whole cell lysate; YAP1, Yes-associated protein 1. 
Alleviation of Collagen Crosslinks Suppresses UUO-Induced Tubular Dilatation and Activation of the ERK/YAP1/Klf5/Cyclin D1 Axis

Our previous study found that collagen crosslinking and deposition were readily detected near the dilated tubules in UUO kidneys, which led to tissue stiffening compared with the nonligated contralateral kidneys (W.-C.C., unpublished data). Blockage of collagen crosslink by BAPN, a lysyl oxidase inhibitor, not only stunted tissue stiffening but also alleviated UUO-induced tubular dilatation, de-differentiation, and EMT. We then evaluated the role of the stiff matrix/ERK/
YAP1/Klf5/cyclin D1 axis in PT cell proliferation of fibrotic kidney induced by UUO (Supplemental Figure S1, A and C). The Western blot analysis results found that mesenchymalrelated markers, $\alpha$-smooth muscle actin and $\beta 1$ integrin, were significantly elevated in lysate from the whole kidney (cortex plus medulla) or cortex part of UUO mice at day 7 . Notably, p-ERK, ERK, Klf5, and cyclin D1 were markedly increased, and Klf4 was decreased in the whole or cortex part of UUO kidneys (Figure 6A). IHC results confirmed the high expression of Klf4 with the no or low expression of p-ERK, ERK, YAP1, Klf5, and cyclin D1 in the kidneys of shamoperated mice (Figure 6B) and contralateral kidneys of UUO

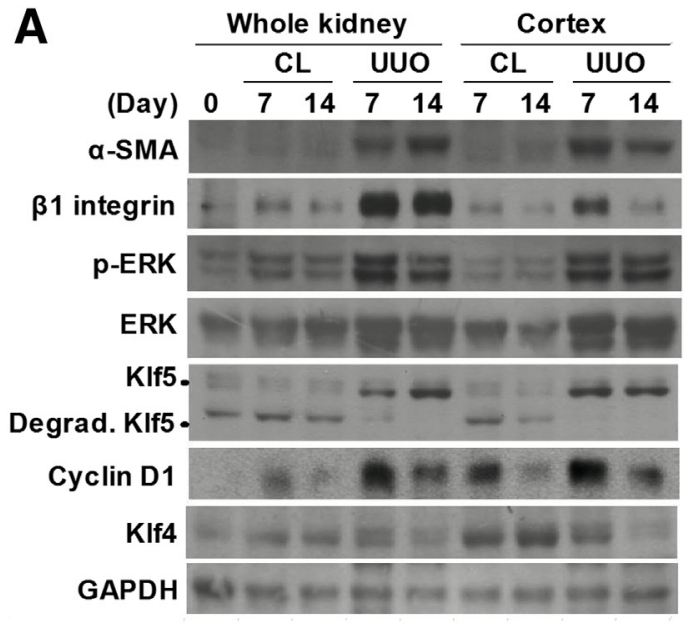

B

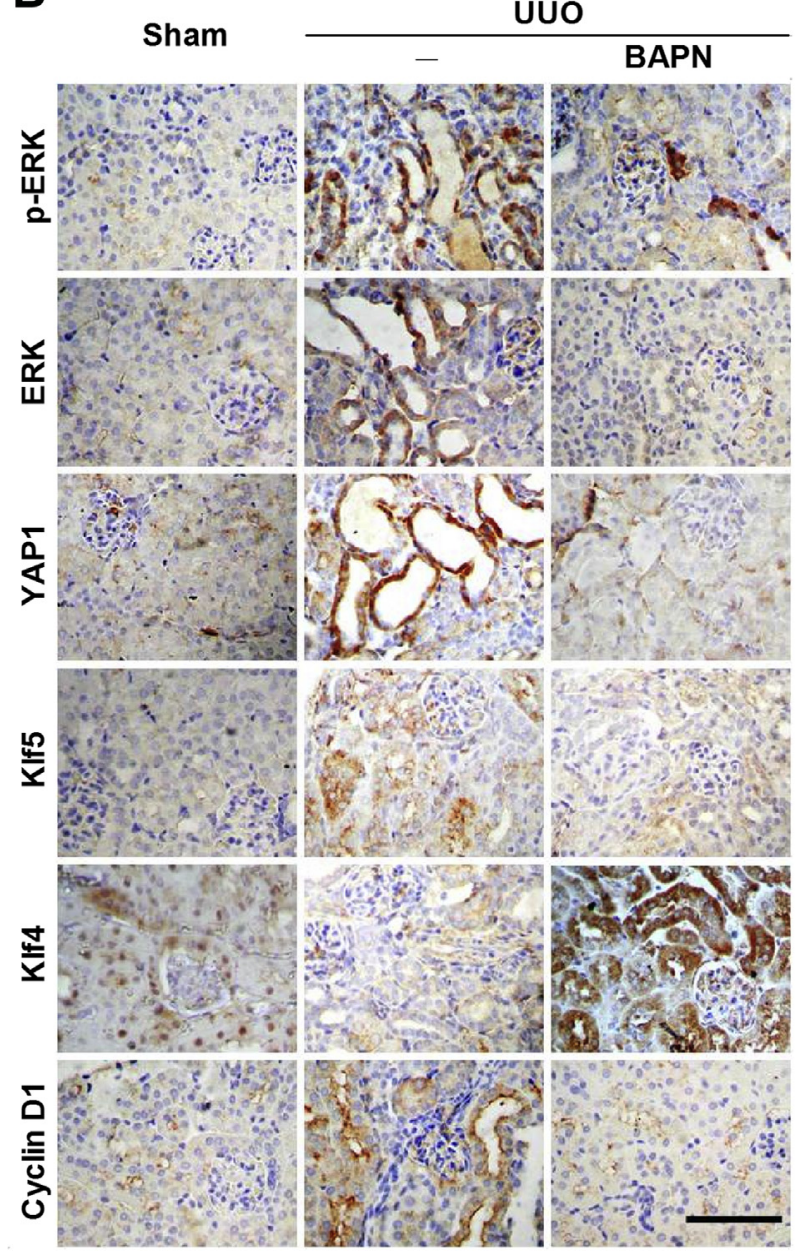

Figure 6 BAPN (a lysyl oxidase inhibitor) attenuates the up-regulation of ERK/YAP1/Klf5/cyclin D1 axis with the down-regulation of Klf4 after UU0. Mice were surgically ligated in the upper region of the left ureteral near the kidney to induce UU0 for 7 and 14 days (Materials and Methods). A: Representative Western blot analysis results of the whole kidney and cortex part from mice without surgery (day 0 ) or with UU0 for the indicated times. The protein levels of $\alpha$-SMA, $\beta 1$ integrin, p-ERK, ERK, Klf5, cyclin D1, and Klf4 were analyzed. GAPDH was used as an internal control. Quantification results of Klf5, Klf4, and cyclin D1 Western blot analyses are shown. GAPDH-normalized data in each condition were compared with those of whole kidney on day 0 (dashed line). B: Representative images show immunohistochemical staining with anti-p-ERK, ERK, YAP1, Klf5, cyclin D1, and Klf4 antibodies of the kidney sections from sham (operated control), UU0, or UUO + BAPN $\left(200 \mathrm{mg} / \mathrm{kg}\right.$ per day via i.p. injection) mice at day $7 .{ }^{*} P<0.05, * * P<0.01$, and ${ }^{* * *} P<0.001 \mathrm{CL}$ versus UU0; ${ }^{\dagger} P<0.05,{ }^{\dagger \dagger} P<0.01$, and ${ }^{\dagger \dagger \dagger} P<0.001 \mathrm{CL}$ or UUO versus normal whole kidney at day 0 . Scale bar $=50 \mu \mathrm{m}$. BAPN, $\beta$-aminopropionitrile; $\mathrm{CL}$, contralateral; Degrad, degraded; ERK, extracellular signal-regulated kinase; GAPDH, glyceraldehyde-3-phosphate dehydrogenase; KIf4, Krüppel-like factor; p-ERK, phosphoextracellular signal-regulated kinase; UU0, unilateral ureteral obstruction; YAP1, Yes-associated protein 1; $\alpha$-SMA, $\alpha$-smooth muscle actin. 
mice (data not shown). After UUO, p-ERK, ERK, YAP1, Klf5, and cyclin D1 were up-regulated in the dilated tubules and enriched in the nucleus, accompanied by the suppression of Klf4 (Figure 6B). BAPN treatment not only preserved Klf4 but also suppressed the increases of ERK/YAP1/Klf5/cyclin D1 in UUO kidneys (Figure 6B). The double staining of Klf5 and aquaporin 1 or proliferating cell nuclear antigen confirmed that BAPN treatment successfully suppressed UUO-elevated Klf5 in proliferative PTs (Supplemental Figure S1, A and C). Taken together, these data suggest that UUO-induced tissue stiffening is critical for the activation of the ERK/YAP1/ Klf5/cyclin D1 axis and the suppression of Klf4, which are relevant to tubular proliferation during renal fibrosis.

\section{Discussion}

Although the contributions of matrix stiffness to cell proliferation and differentiation are increasingly understood, little is known about the functional relation between matrix stiffness and TFs. In this study, we report that matrix stiffness-affected cell proliferation is relevant to the inverse expression of Klf5 and Klf4 (Figures 3 and 4). Soft matrix induces low levels of Klf5 and high levels of Klf4, which cause growth arrest. In contrast, stiff matrix induces high levels of Klf5 and low levels of Klf4, which promote mPTEC proliferation. Such regulation is also observed in vivo. Low levels of Klf5 and high levels of Klf4 were detected in the PTs of normal kidneys (Figure 6 and Supplemental Figure S1, A and C). After UUO, the inverse expression of Klf5 and Klf4 were switched with highly expressed cyclin D1. Inhibition of lysyl oxidase by BAPN not only lessened UUO-induced collagen crosslinking and fibrosis (W.-C.C., unpublished data) but also prevented UUO-induced inverse expression of Klf5 and Klf4 with the increase in cyclin D1 (Figure 6B). Through a combination of ex vivo and in vivo analyses, we suggest that matrix stiffness plays a critical role in regulating the inverse expression of Klf5 and Klf4 in PTs. Previously, Fujiu et al ${ }^{32}$ found that Klf5 is mainly expressed in collecting duct cells in the normal mouse kidney and contributes to the inflammatory responses to UUO. However, whether matrix stiffness also contributed to other renal cell proliferation in a Klf5-dependent manner is unclear. Notably, we found that the level of Klf5 positively correlated with the level of cyclin D1, both decreased with decreasing matrix stiffness in PTs, distal tubules, and collecting duct cells but not in endothelial cells or fibroblasts (Supplemental Figure S1, E and F). Whether these cells shared the similar TF regulation in matrix stiffness-driven cell proliferation needs to be confirmed.

Reports found that mechanical cues from cell density, geometry, or physical stimulus (stretch or matrix stiffness) are transduced by two transcriptional co-activators, YAP and TAZ (transcriptional coactivator with PDZ-binding motif). ${ }^{50}$ Camargo et $\mathrm{al}^{51}$ reported that YAP1 translated the distribution of spatial force into pattern growth within multicellular layers of mammary glands. ${ }^{52}$ Here, we report that YAP1 was activated in cells located at the basal region of multicellular
mPTECs, suggesting the mechanical cues were spatially distributed to regulate cell proliferation (Figure 5, B-D). Moreover, soft matrix stunted YAP1 activation. Collectively, cells adjacent to a stiff matrix receive the highest mechanical cues and display well-organized stress fibers (Supplemental Figure S2C) and nuclear YAP1 (Figure 5, B-D). In the in vivo study, UUO-induced YAP1 expression and nuclear translocation were abolished by BAPN, thus highlighting the importance of matrix stiffness in the regulation of YAP1 activity (Figure 6B).

Klf4 and Klf5 were reported to antagonize each other in controlling expression of cyclin D1 by binding to the sp1 motif on the cyclin D1 promoter. ${ }^{2,53}$ Our data confirm that Klf5 increased cyclin D1 expression, whereas Klf4 repressed it (Figure 4). The expression of Klf5 is mechanoresponsive, and stiff matrix thus increased the amount of Klf5 protein through both transcriptional regulation and post-translational regulation (Figures 2C and 3C). Post-translational modifications, including acetylation, phosphorylation, and sumoylation, were reported to regulate Klf5 stability, transcription activity, binding affinity, and nuclear translocation. ${ }^{54-57}$ Notably, direct binding by YAP1 stabilized Klf5 in the nucleus and prevented its degradation. ${ }^{48}$ Here, we found that stiff matrixincreased nuclear YAP1 tightly correlated with the spatially restricted patterns of Klf5 and cyclin D1 in the MPTEC aggregate. We therefore propose that stiff matrix promotes cell proliferation through activating YAP1, which relays a mechanical cue to enhance cyclin D1 expression by stabilizing Klf5.

Soft matrix suppressed not only cell proliferation but also transformation growth factor- $\beta 1-$ induced EMT. ${ }^{12}$ In addition to serving transcriptional repressor for cyclin D1, Klf4 also functions as a transcriptional activator of epithelial genes and as a repressor of mesenchymal genes. Soft matrixpreserved Klf4 should thus be emphasized to maintain phenotype differentiation and growth arrest. Once matrix stiffness is increased, the mechanoresponsive YAP1 is activated, which subsequently increases the level of Klf5. Dang et $\mathrm{al}^{14}$ reported that Klf4 and Klf5 exert opposing effects on the promoter of the Klf4 gene by physical competition. Klf4 activates the promoter of its own gene, and Klf5 suppresses the Klf4 promoter. In addition, Klf4 abrogates the inhibitory effect of Klf5 on the Klf4 promoter, and Klf5 abrogates the activating effect of Klf4 on the same promoter. We thus suggest that the inverse expression of Klf5 and Klf4 is determined by mechanical cue-regulated Klf5.

In summary, we verified the novel mechanism of mechanical cue from stiff matrix that induced cell proliferation and its significance related to the pathogenesis of renal fibrosis. Mechanical cues, as transduced by YAP1, regulate the inverse expression of Klf5 and Klf4 to determine cell proliferation. Thus, the maintenance of physiologic tissue stiffness thus turns out to be crucial for organ homeostasis. Inhibition of Klf5 increase/Klf4 decrease may provide insights for developing antifibrotic therapies via alleviating tissue stiffening. 


\section{Acknowledgments}

We thank Drs. Yang-Kao Wang, Chia-Ching Wu, and Yi-Chao Lee for helpful suggestion on the manuscript and Dr. Yi-Chun Yeh, Hsiu-Kuan Lin, Chia-Yu Chang, I-Hsuan Lin, and Tzu-Ling Chen for technical assistance.

W.-C.C. initiated and performed all of the experiments; W.-C.C., H.-H.L., and M.-J.T. conceived the study and wrote the manuscript.

\section{Supplemental Data}

Supplemental material for this article can be found at http://dx.doi.org/10.1016/j.ajpath.2015.05.019.

\section{References}

1. Engler AJ, Sen S, Sweeney HL, Discher DE: Matrix elasticity directs stem cell lineage specification. Cell 2006, 126:677-689

2. Paszek MJ, Zahir N, Johnson KR, Lakins JN, Rozenberg GI, Gefen A, Reinhart-King CA, Margulies SS, Dembo M, Boettiger D, Hammer DA, Weaver VM: Tensional homeostasis and the malignant phenotype. Cancer Cell 2005, 8:241-254

3. Wang YH, Chiu WT, Wang YK, Wu CC, Chen TL, Teng CF, Chang WT, Chang HC, Tang MJ: Deregulation of AP-1 proteins in collagen gel-induced epithelial cell apoptosis mediated by low substratum rigidity. J Biol Chem 2007, 282:752-763

4. Li Z, Dranoff JA, Chan EP, Uemura M, Sevigny J, Wells RG: Transforming growth factor-beta and substrate stiffness regulate portal fibroblast activation in culture. Hepatology 2007, 46:1246-1256

5. Janmey PA, Miller RT: Mechanisms of mechanical signaling in development and disease. J Cell Sci 2011, 124:9-18

6. Dobrev HP: In vivo study of skin mechanical properties in patients with systemic sclerosis. J Am Acad Dermatol 1999, 40: 436-442

7. Timar O, Soltesz P, Szamosi S, Der H, Szanto S, Szekanecz Z, Szucs G: Increased arterial stiffness as the marker of vascular involvement in systemic sclerosis. J Rheumatol 2008, 35:1329-1333

8. Seewaldt V: ECM stiffness paves the way for tumor cells. Nat Med 2014, 20:332-333

9. Georges PC, Hui JJ, Gombos Z, McCormick ME, Wang AY, Uemura M, Mick R, Janmey PA, Furth EE, Wells RG: Increased stiffness of the rat liver precedes matrix deposition: implications for fibrosis. Am J Physiol Gastrointest Liver Physiol 2007, 293: G1147-G1154

10. Song ZZ: Acute viral hepatitis increases liver stiffness values measured by transient elastography. Hepatology 2008, 48:349-350

11. Meng XM, Chung AC, Lan HY: Role of the TGF-beta/BMP-7/Smad pathways in renal diseases. Clin Sci (Lond) 2013, 124:243-254

12. Chen WC, Lin HH, Tang MJ: Regulation of proximal tubular cell differentiation and proliferation in primary culture by matrix stiffness and ECM components. Am J Physiol Renal Physiol 2014, 307:F695-F707

13. Suske G, Bruford E, Philipsen S: Mammalian SP/KLF transcription factors: bring in the family. Genomics 2005, 85:551-556

14. Dang DT, Zhao W, Mahatan CS, Geiman DE, Yang VW: Opposing effects of Kruppel-like factor 4 (gut-enriched Kruppel-like factor) and Kruppel-like factor 5 (intestinal-enriched Kruppel-like factor) on the promoter of the Kruppel-like factor 4 gene. Nucleic Acids Res 2002, 30:2736-2741

15. McConnell BB, Ghaleb AM, Nandan MO, Yang VW: The diverse functions of Kruppel-like factors 4 and 5 in epithelial biology and pathobiology. BioEssays 2007, 29:549-557
16. Ghaleb AM, Nandan MO, Chanchevalap S, Dalton WB, Hisamuddin IM, Yang VW: Kruppel-like factors 4 and 5: the yin and yang regulators of cellular proliferation. Cell Res 2005, 15:92-96

17. Ema M, Mori D, Niwa H, Hasegawa Y, Yamanaka Y, Hitoshi S, Mimura J, Kawabe Y, Hosoya T, Morita M, Shimosato D, Uchida K, Suzuki N, Yanagisawa J, Sogawa K, Rossant J, Yamamoto M, Takahashi S, Fujii-Kuriyama Y: Kruppel-like factor 5 is essential for blastocyst development and the normal self-renewal of mouse ESCs. Cell Stem Cell 2008, 3:555-567

18. Nandan MO, Yoon HS, Zhao W, Ouko LA, Chanchevalap S, Yang VW: Kruppel-like factor 5 mediates the transforming activity of oncogenic H-Ras. Oncogene 2004, 23:3404-3413

19. Sun R, Chen X, Yang VW: Intestinal-enriched Kruppel-like factor (Kruppel-like factor 5) is a positive regulator of cellular proliferation. J Biol Chem 2001, 276:6897-6900

20. Nandan MO, Chanchevalap S, Dalton WB, Yang VW: Kruppel-like factor 5 promotes mitosis by activating the cyclin B1/Cdc2 complex during oncogenic Ras-mediated transformation. FEBS Lett 2005, 579:4757-4762

21. Shields JM, Christy RJ, Yang VW: Identification and characterization of a gene encoding a gut-enriched Kruppel-like factor expressed during growth arrest. J Biol Chem 1996, 271:20009-20017

22. Katz JP, Perreault N, Goldstein BG, Lee CS, Labosky PA, Yang VW, Kaestner KH: The zinc-finger transcription factor Klf4 is required for terminal differentiation of goblet cells in the colon. Development 2002, 129:2619-2628

23. Segre JA, Bauer C, Fuchs E: Klf4 is a transcription factor required for establishing the barrier function of the skin. Nat Genet 1999, 22:356-360

24. Yu T, Chen X, Zhang W, Li J, Xu R, Wang TC, Ai W, Liu C: Kruppel-like factor 4 regulates intestinal epithelial cell morphology and polarity. PLoS One 2012, 7:e32492

25. Swamynathan SK, Davis J, Piatigorsky J: Identification of candidate Klf4 target genes reveals the molecular basis of the diverse regulatory roles of Klf4 in the mouse cornea. Invest Ophthalmol Vis Sci 2008, 49:3360-3370

26. Yori JL, Johnson E, Zhou G, Jain MK, Keri RA: Kruppel-like factor 4 inhibits epithelial-to-mesenchymal transition through regulation of E-cadherin gene expression. J Biol Chem 2010, 285:16854-16863

27. Shimizu Y, Takeuchi T, Mita S, Notsu T, Mizuguchi K, Kyo S: Kruppel-like factor 4 mediates anti-proliferative effects of progesterone with G0/G1 arrest in human endometrial epithelial cells. J Endocrinol Invest 2010, 33:745-750

28. Chen X, Johns DC, Geiman DE, Marban E, Dang DT, Hamlin G, Sun R, Yang VW: Kruppel-like factor 4 (gut-enriched Kruppel-like factor) inhibits cell proliferation by blocking G1/S progression of the cell cycle. J Biol Chem 2001, 276:30423-30428

29. Shie JL, Chen ZY, Fu M, Pestell RG, Tseng CC: Gut-enriched Kruppel-like factor represses cyclin D1 promoter activity through Sp1 motif. Nucleic Acids Res 2000, 28:2969-2976

30. Takahashi K, Yamanaka S: Induction of pluripotent stem cells from mouse embryonic and adult fibroblast cultures by defined factors. Cell 2006, 126:663-676

31. Yu F, Li J, Chen H, Fu J, Ray S, Huang S, Zheng H, Ai W: Kruppel-like factor 4 (KLF4) is required for maintenance of breast cancer stem cells and for cell migration and invasion. Oncogene 2011, 30:2161-2172

32. Fujiu K, Manabe I, Nagai R: Renal collecting duct epithelial cells regulate inflammation in tubulointerstitial damage in mice. J Clin Invest 2011, 121:3425-3441

33. El-Dahr SS, Aboudehen K, Saifudeen Z: Transcriptional control of terminal nephron differentiation. Am J Physiol Renal Physiol 2008 294:F1273-F1278

34. Saifudeen Z, Dipp S, Fan H, El-Dahr SS: Combinatorial control of the bradykinin B2 receptor promoter by p53, CREB, KLF-4, and CBP: implications for terminal nephron differentiation. Am J Physiol Renal Physiol 2005, 288:F899-F909

35. Hayashi K, Sasamura H, Nakamura M, Azegami T, Oguchi H, Sakamaki Y, Itoh H: KLF4-dependent epigenetic remodeling 
modulates podocyte phenotypes and attenuates proteinuria. J Clin Invest 2014, 124:2523-2537

36. Edgar R, Domrachev M, Lash AE: Gene Expression Omnibus: NCBI gene expression and hybridization array data repository. Nucleic Acids Res 2002, 30:207-210

37. Yeh YC, Wei WC, Wang YK, Lin SC, Sung JM, Tang MJ: Transforming growth factor-\{beta 1 induces Smad3-dependent $\{$ beta 1 integrin gene expression in epithelial-to-mesenchymal transition during chronic tubulointerstitial fibrosis. Am J Pathol 2010, 177: $1743-1754$

38. Wu MJ, Wen MC, Chiu YT, Chiou YY, Shu KH, Tang MJ: Rapamycin attenuates unilateral ureteral obstruction-induced renal fibrosis. Kidney Int 2006, 69:2029-2036

39. Lee PT, Lin HH, Jiang ST, Lu PJ, Chou KJ, Fang HC, Chiou YY, Tang MJ: Mouse kidney progenitor cells accelerate renal regeneration and prolong survival after ischemic injury. Stem Cells 2010, 28:573-584

40. Wei WC, Lin HH, Shen MR, Tang MJ: Mechanosensing machinery for cells under low substratum rigidity. Am J Physiol Cell Physiol 2008, 295:C1579-C1589

41. Yeh YC, Wu CC, Wang YK, Tang MJ: DDR1 triggers epithelial cell differentiation by promoting cell adhesion through stabilization of E-cadherin. Mol Biol Cell 2011, 22:940-953

42. Suzuki K, Bose P, Leong-Quong RY, Fujita DJ, Riabowol K: REAP: a two minute cell fractionation method. BMC Res Notes 2010, 3:294

43. Chiou YW, Lin HK, Tang MJ, Lin HH, Yeh ML: The influence of physical and physiological cues on atomic force microscopy-based cell stiffness assessment. PLoS One 2013, 8:e77384

44. Zhang XH, Zheng B, Han M, Miao SB, Wen JK: Synthetic retinoid Am80 inhibits interaction of KLF5 with RAR alpha through inducing KLF5 dephosphorylation mediated by the PI3K/Akt signaling in vascular smooth muscle cells. FEBS Lett 2009, 583:1231-1236

45. Du JX, Hagos EG, Nandan MO, Bialkowska AB, Yu B, Yang VW: The E3 ubiquitin ligase SMAD ubiquitination regulatory factor 2 negatively regulates Kruppel-like factor 5 protein. J Biol Chem 2011, 286:40354-40364

46. Chen C, Zhou Z, Guo P, Dong JT: Proteasomal degradation of the KLF5 transcription factor through a ubiquitin-independent pathway. FEBS Lett 2007, 581:1124-1130
47. Chen C, Sun X, Ran Q, Wilkinson KD, Murphy TJ, Simons JW, Dong JT: Ubiquitin-proteasome degradation of KLF5 transcription factor in cancer and untransformed epithelial cells. Oncogene 2005, 24:3319-3327

48. Zhi X, Zhao D, Zhou Z, Liu R, Chen C: YAP promotes breast cell proliferation and survival partially through stabilizing the KLF5 transcription factor. Am J Pathol 2012, 180:2452-2461

49. Halder G, Dupont S, Piccolo S: Transduction of mechanical and cytoskeletal cues by YAP and TAZ. Nat Rev Mol Cell Biol 2012, 13:591-600

50. Dupont S, Morsut L, Aragona M, Enzo E, Giulitti S, Cordenonsi M, Zanconato F, Le Digabel J, Forcato M, Bicciato S, Elvassore N, Piccolo S: Role of YAP/TAZ in mechanotransduction. Nature 2011, 474: 179-183

51. Camargo FD, Gokhale S, Johnnidis JB, Fu D, Bell GW, Jaenisch R, Brummelkamp TR: YAP1 increases organ size and expands undifferentiated progenitor cells. Curr Biol 2007, 17:2054-2060

52. Aragona M, Panciera T, Manfrin A, Giulitti S, Michielin F, Elvassore N, Dupont S, Piccolo S: A mechanical checkpoint controls multicellular growth through YAP/TAZ regulation by actinprocessing factors. Cell 2013, 154:1047-1059

53. Suzuki T, Sawaki D, Aizawa K, Munemasa Y, Matsumura T, Ishida J, Nagai R: Kruppel-like factor 5 shows proliferation-specific roles in vascular remodeling, direct stimulation of cell growth, and inhibition of apoptosis. J Biol Chem 2009, 284:9549-9557

54. Matsumura T, Suzuki T, Aizawa K, Munemasa Y, Muto S, Horikoshi M, Nagai R: The deacetylase HDAC1 negatively regulates the cardiovascular transcription factor Kruppel-like factor 5 through direct interaction. J Biol Chem 2005, 280:12123-12129

55. He M, Han M, Zheng B, Shu YN, Wen JK: Angiotensin II stimulates KLF5 phosphorylation and its interaction with c-Jun leading to suppression of p21 expression in vascular smooth muscle cells. J Biochem 2009, 146:683-691

56. Zhang Z, Teng CT: Phosphorylation of Kruppel-like factor 5 (KLF5/IKLF) at the CBP interaction region enhances its transactivation function. Nucleic Acids Res 2003, 31:2196-2208

57. Du JX, Bialkowska AB, McConnell BB, Yang VW: SUMOylation regulates nuclear localization of Kruppel-like factor 5. J Biol Chem 2008, 283:31991-32002 